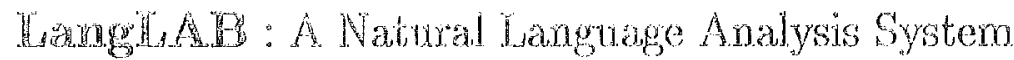

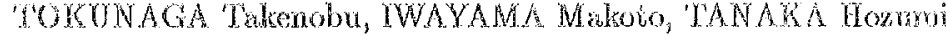 \\ Departwent of Comproter Science \\ Tokyo Thstitute of Technology
}

\author{
KAMTWAKY Tadashi \\ Mitach Teseard labomatory \\ Hitsehi Itid.
}

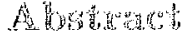

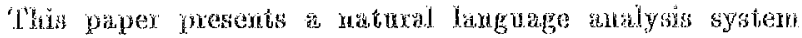

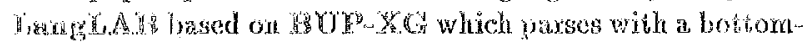
ap and dephinst strategy and has abiling to handlo loft axhaposition. We have altudy developed a grammar for-

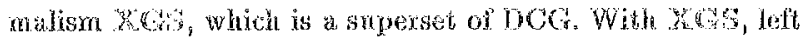

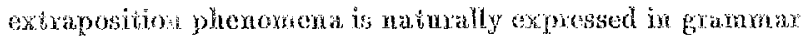

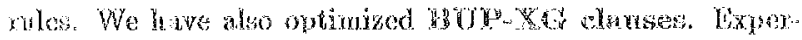
iments showed that in comparison to the original tat xer systen, the wnalysis aped up 10 times in the interpecer

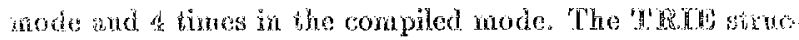

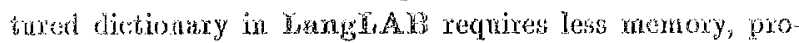
vides faster dictonary reterence and also Itandles complcated idioms with versatility. Consequently, the atilightion

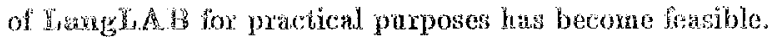

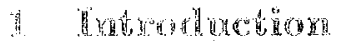

So thr, sevenal grommax fomalisin based on logic progran ming parndign such as lihetanorphosis Crammax [2] and DCC [: each grammar rule is translated into a. Dorn clause, and the Prolog interoreter parses the input wertence with these form

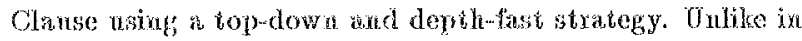
the past where parsers had to be constronted for syntuctic analygis, in this nxethod, we do not have to becanse the pro. log interneter itself works as one. Metamorphosis Grammar also provides is natural language processing method which interleaves syntactic analysis and semantic analysis. This is a desirable feature from the point of view of cognitive acience.

Tollowing Metamorphosis Grammar, pereira at al, developed a grommat fomalism called Definite Chase Gram-

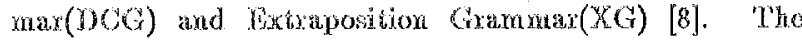
gramarar no les written in $9 \mathrm{CG}$ are also translated into a Prolog program and the Prolog interpreter works as a top dow and depth-inst pansen interleaving syatax antysis and whementio analysis. XCr is the extended version of DCO cupable of handing let exiraposition.

Towever, bog-down parsen have a problem thathe pro-

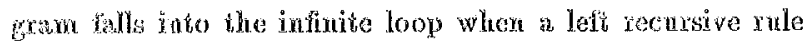
appears in the grammar mos. This problem sou bo solved

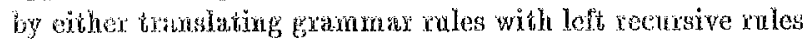

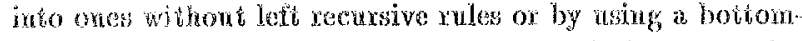

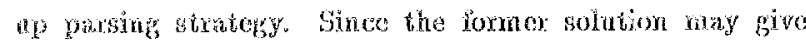

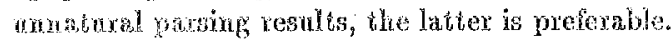

Matsurobo of Alectotechnical Laboratory developed in system in which the grammates written in DCG we tranglated no Mon dauses whled BOP clauses and Pro-

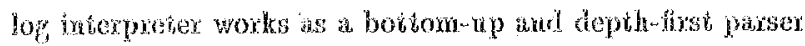

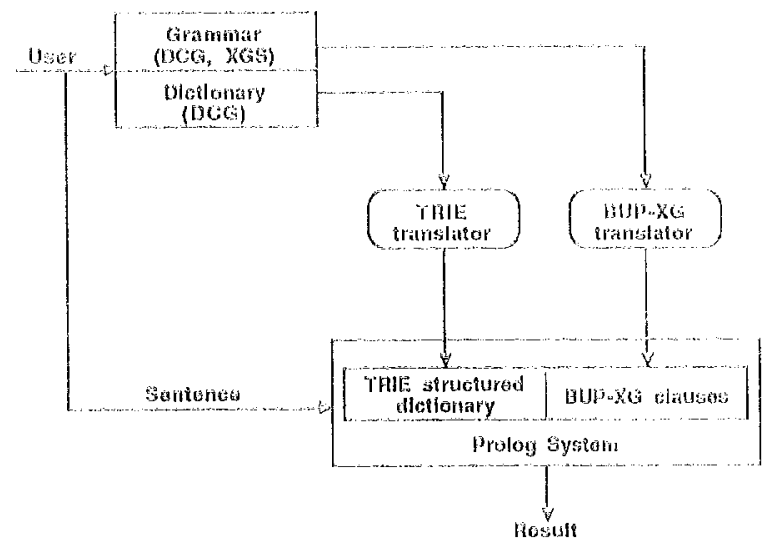

Figure 1: Strmcture of LangLAls

for thesn rules [14]. Matsumotos: system is called the BOP system. The BOF sysien can handle left recusipe rulos and, bat grammar mes mod the dictionary separaty.

Konno of Tokyo Institute of Technology extended the BOP system to BOP-XG system [5] which cain handle the lett extraposition phenomena elegantly. BUP-XG system introduced the grammat description form called XCS ( $\mathrm{CX}$ iraposition Grammar with Slash Category).

'This paper preseats a natural language aralysis system LanglaA based on Konno's BUP.XY system. Figme 1 shows the structure of the Jarigl,AB system. Users should prepare grammar rules written in XGS and a dictionary written in DCG. Both grammar rules and a dictionary ane Exansluted into BOP-XG clauses and TrRe structured dictionary respectively by translators. Translated results are consulted by the Prolog system and the Prolog interpreter works as a parser.

In chapter 2, we briefly explain the fondmmentals of the BOP system and the grammar description form XCS adopted in LanglAB. We will also describe BOP-XG trantio lator which traxishtes the grammax written in XCS into BUJ XG clause and its optimizations. In chapter 3 , we will towh on the 'PRME stractured dictionary adopted in Lang,LAB. 'TRIT structured dictionary requires less menory and provides faster dichonaly reterenco and provides llexible idiont handhing. In chapter: 4 , we shall present iosults of experiments verifying the eficect of the optimization described in chapter 2. Experinents showed that the andel. ysis aped np 10 times in the interpretive mode and 4 times in the compiled mode. The authom believe that Jamgl,AB perforns well enough to bo of practical use. 


$$
\begin{aligned}
& \text { s } \quad \cdots \rightarrow \text { np, vp. } \quad(d-i) \\
& \text { mp a..> pron. } \quad(d-2) \\
& \text { pron } \rightarrow \text { [you]. } \quad(d-3) \\
& \text { vp } \rightarrow \rightarrow \text { [rálk]. } \quad(d \sim 4)
\end{aligned}
$$

Fignre 2: Sample grammar written in DCG

$$
\begin{aligned}
& n p(G) \rightarrow\{\operatorname{Link}(n p, G)\}, \\
& \text { goal (vp), } \\
& s(G) \text {. } \\
& \operatorname{pron}(G) \rightarrow \operatorname{mp}(G) . \quad(b-2) \\
& \text { dict (pron) } \rightarrow-\infty \text { [you]. } \quad(b-3) \\
& \operatorname{aict}(r p) \rightarrow \text { [ralk]. (b-4) }
\end{aligned}
$$

Figure 3: BUP clanses translated from figure 2

\section{$2 \mathrm{XGS}$ and $\mathrm{BU} \mathrm{P}-\mathrm{XG}$}

In this chapter, we shall explain the grammar description form XGS adopted in LangLAB and the BUP-XG translator which translates grammar rules writton in XGS into the BUP-XG clauses. Before explaining BUP-XG, we will briefly explain the mechanism of the BUP system, the predecessor of BUP-XG. Basic parsing mechanism of BUP is left-corner parsing with top-down prediction.

\subsection{BUP system}

In BUP system, grammar rules written in DCG (Figure 2) arc translated into the rules called BUP clauses which are also of DCG format, and some Prolog program (link clauses and termination clauses : explained later).

Fignre 3 shows results of the translation. These BUP clanses are then translated into a Prolog program (ligure 4) by the DCG translator which is embedded in the Prolog system. Two more arguments are added to each predicate which denotes nonterminal symbol in figure 4. These arguments constitutes a difference list which represents the input string. With the special predicate goal which is necessary for bottom up parsing, this Prolog program can parse the input string with a bottom-up and depth-first strategy. Figure 5 shows the definition of the predicate goal.

Now, we shall give a step by step explanation of the parsing algorithm of the BUP system. We will use the grammar shown in figure 4 and input sentence "you walk" as an example. Calling the predicate goal activates the parsing process:

$$
\begin{aligned}
& \text { ?- goal (s, [you, palk], []). } \\
& \begin{array}{ll}
\operatorname{np}(G, X, Z): \cdots \operatorname{link}(n p, G), & (p-1) \\
\quad \operatorname{goal}(\vee p, X, Y), & \\
& s(G, Y, Z) . \\
\operatorname{pron}(G, X, Y):-n p(G, X, Y) . & (p-2) \\
\operatorname{dict}(\operatorname{pron},[\text { you } \mid X], X) . & (p-3) \\
\operatorname{dict}(v p,[\text { ral } \mid X], X) . & (p-4)
\end{array}
\end{aligned}
$$

Figure 4: Prolog programs translated from figuxe 3

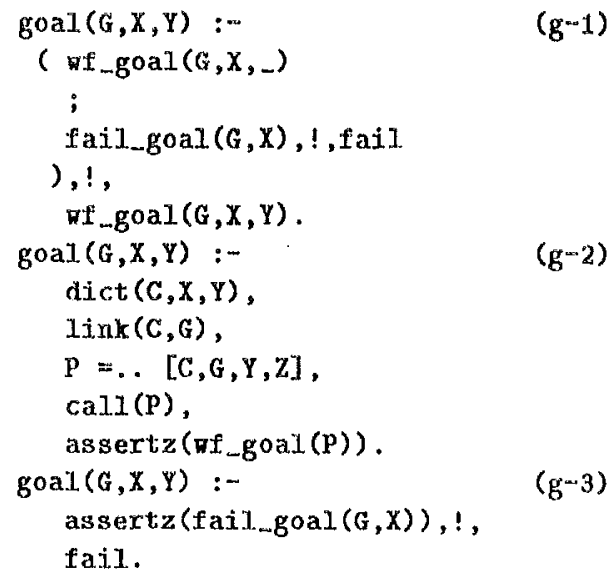

Figure 5: Definition of the goal clause

This calling checks to see if :

A parse tree the root of which is the category " $\mathrm{s}$ ", can be constructed from the input string denoted by the difference between the list [you, walk] and the list [ ] ([you, palk] in this example).

The first call of goal invokes the clause $(\mathrm{g}-1)$ in the figure 5 . The clause $(g-1)$ checks to see if the same analysis have been made before, to avoid recomputation using the information previously asserted as wf_goal and fail_goal.

As the execution of the clause $(g-1)$ fails in this case, the system chooses the next clause $(g-2)$. In the body of the clause $(g-2)$, the system consults the dictionary by calling "dict (C, [you, walk], Y)". This predicate call picks ( $\mathrm{p}-3$ ) in figure 4 and the system matches "pron" with variable $\mathrm{C}$ and "[walk]" with variable $Y$.

In the second line of $(\mathrm{g}-2)$, the system calls the predicate link to see if the category which is obtained by the previous dictionary consultation ("pron" in this example) can be leftcorner of the current goal (" $\mathrm{s}$ " in this example). The link clauses are calculated by the BUP translator. Suppose this test succeeds, the system calls the predicate "pron" :

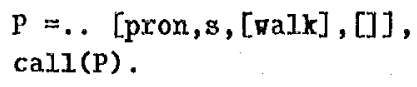

Calling "pron ( $s,[$ walk],[] )" invokes $(p-2)$. Then, the system executes its body that is, "np (s, [walk], [1)".

Calling " $n p(s,[$ wa.tx], $[$.$] )" invokes the clause (p-1)$. After calling the predicate link to check a reachability from "np" to "s", the system invokes "goal (vp, [ra.lk], [])". At this point, the system has analyzed the string "you" as "np" and is predicting that the trailing string "walk" should be bundled up to the category "vp".

In the same manner, a bottom-up analysis with a topdown prediction proceeds until the execution of goal with the termination clauses succeeds. See [14] for the detail of the termination clauses.

Results once succeeded or failed in an analysis are asserted as wf goal in the end of $(\mathrm{g}-2)$ and fail_goal in the clause $(\mathrm{g}-3)$ respectively. This information is used in $(\mathrm{g}-1)$ as described. 


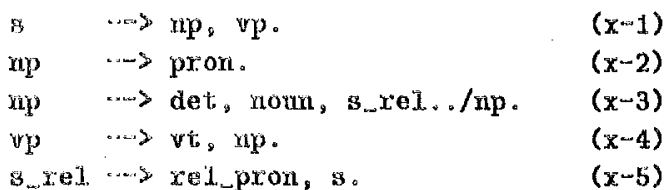

Pigure 6: Sample grammar written in XGS

\subsection{BUP-XG system}

The embedded sentence which appears in relative clanses in Tinglish can be viewed as a structure in which a noun phrase is missing from declarative sortence. A gap is formed as a result of moving the antecedent from within the declarative senterce to the left of the relative clause. Linguists call such phenomexta "Left extraposition". By considering the gap left by the mowal constitments as a "trace", and incorporating a mechanism that looks for such a "trace" automatically, the number of grammar rules can be decreased and the gramum rules becoms easier to read. Moreover incorporating such mechanism contributes to making analysis speed faster.

Top-down paxsers like ATNG [13], [12] and XG [8] incorporate such a mechanism. The top-down parser can predict what categocy the trailing input string may be bundled up to. Efficient trace searching is possible as the system assumes the existence of traces only when a particular category is predicted as a goal.

A pure botitom-up parser is not capable of such predictions and inefficieacy results because of the necessity to assume the existence of a trace between every two words. However, since the BVP system incorporates top-down prediction in the bottom-ap parsing strategy described in 2.1, it is possible to implement the mechanism to look for the traces efficiently. Konno developed a BUP-XG system which incorporated such a mechanism [5].

The XGS adopted in LangLAB provides grammar writers the facility with which left extraposition can be naturally expressed in grammar rules. Figure 6 shows a small Linglish grammar wlich is written in XGS.

The notation ". .f" (called "slash") in the rule $(x-3)$ is introduced in XGS. This rule means that there exists the syntactic category "np" which dominates the "trace" under the syntactic category "s_rel" ("s_rel" means relative sentence). This idea is influenced by the "slash category" in GPSG [3]. We call the category after "../" "slash category". Rule $(x-3)$ also shows that the category "np" consists of the categories "det", "noun" and "s_rel" and that the trace left behind by the left extraposition of the noun phrase consisting of "det" and "noun" is dominated by "s.rel". During an analysis, when the system finds the trace under "s.rel", as shown in ftgure 7 , its associates the trace in the embedded sentence with the moved phrase ("the man").

XQXS also provides a notation to represent "Ross's Complex NP constraint" [10]. Following is an example of this notation. "Chis notation is called "open $(<)$ " and "close (s)" following Pereira [8].

$$
a \cdots>b, c,\langle d\rangle \text {. }
$$

This xule mans that category "a" consists of categories " $b$ ", "c" and " $d$ ". Open-close notation defines the scope of extra-

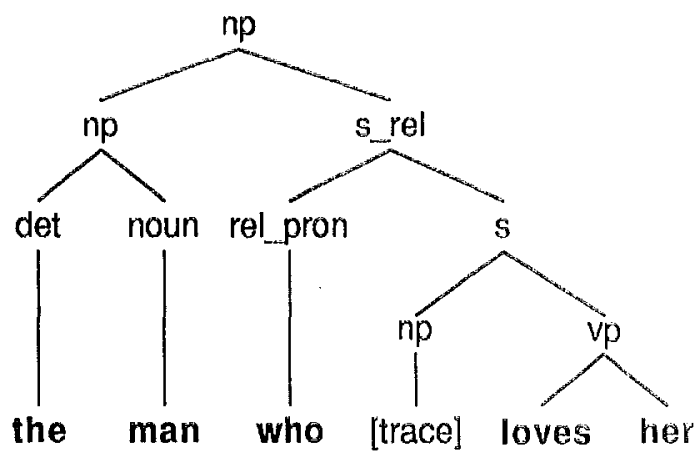

Figure 7: Matching between slash category and its trace

position. This example says that the movement from under "b" or " $\mathrm{c}$ " to the outside of " $\mathrm{a}$ " is permissible, but the movement from under "d" to the outside of "a" is not. Sentences violating "Ross's Complex $N$ p constraint" are rejected by modifying $(x-3)$ to become $\left(x-3^{\prime}\right)$

$$
\text { np } \rightarrow \text { det, noun, }\left\langle s_{-} \text {re1../np }\right\rangle .\left(x^{-3} 3^{\prime}\right)
$$

With $\left(x-3^{\prime}\right)$, the trace which is dominated by slash category "np" under "s.rel" can only correspond to the noun phrase which consists of "det" and "noun".

In addition, XGS also provides a double arrow notation $(=\Rightarrow)$ and the notation to describe $X$ lists (explained later) explicitly. With these notation, "coordinate structure" can be represented in a natural way (see [5]).

\subsection{BUP-XG translator}

Just like the BUP system, the grammar rules written in $X$ GS are translated into BUP-XG clauses, link clauses and termination clauses by the BUP-XG translatol. The BUP$X G$ translator in the TangL $\triangle B$ system has been improved so as to generate BUP-XG clauses more optimized than that in the original BUP-XG system. Furthermore, it is also equipped with a new function which inserts purse tree information automatically. 'The translator takes about three seconds to translate a grammar of about 200 rules. The following subsection explains these improvements.

\subsubsection{Representation of link clauses}

As the number of grammar rules increases, more link clauses are generated by the translator. For example, from about 200 grammar rules of English which we have developed, the BUP-XG translator generates about 700 link clanses. Shortening the search time of link clanses wonld contribute to an efficient analysis.

Link clauses are called in the body of BUP-X.G clauses and in the predicate goal. Since both the arguments of link are atoms in the both cases, a link

$\operatorname{link}(a, b)$.

which denotes the reachablity from the category " $a$ " to " $b$ " can be change to the form

$$
a(b):-! \text {. }
$$

This form of representation reduces the search space of the reachablity test. The $\mathrm{BUP}-\mathrm{XG}$ translator in LangLAB generates link information of this form. 


\subsubsection{Tuleres for difyernee list}

As desuribed in subsection 2.1 input string are represented by a difference list and intermediate analysis results are as.serted with the predicate wr goal and fuil goal. Since the last two arguments of the wr fogal constitutes a differonce list of the input string, the longer the input string becones, the more mexnory wit goal consumes. By indexing differ. ence lists, the amount of memory required is reduced, and faster reference to intermediate results is possible.

lior example, when the system gets the input siring "you walk", the predicates text are asserted as follows:

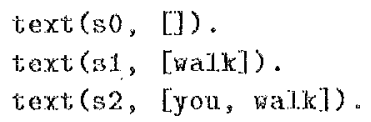

The dictionary reference program gets a difference list by calling text with indexes $(s 1,52, \ldots)$ as the key, before consulting the dictionary.

\subsection{Representation of intermediate results}

Generally, a long input string gives rise to more wf gonls and fail goals which results in longer search time for intermediate analysis results. Wf gonls and fail goals have as their arguments, the index to the dificrence list denoting the partial input string, and its analysis. As described in 2.1; goal first consults wif gouls and fril_gonls with the indexes of input string as the key. In LangLAB system, the predicate names of intermediate analysis result are the indexes to the difference list instead of "wi_goal." or "fail_goal". "This modification reduces the senrch space of the intemediate analysis results and speeds up the analysis process.

\subsubsection{Insertion of parse tree information}

Users sometirnes require the results of symtactic analysis to be expressed as parse trees, and in both the BUP systern and the original BUP $\mathrm{XG}$ system, users are required to insert ax argument in each category to accommodate parse tree information. Howcver, it is not a difficult task to make the translator insert this information automatically. In the $B O P-X C$ translator of Jangl.AB, this information is inserted automatically unless instructed otherwise. This function is similax to the one in the McCord's MLG(Modular Logic Grammar) [\%]. However, mlike MLC, all the nonterminal symbols can be a node of parse trees.

\subsubsection{Example of trinslation}

Figure 8 shows the $B O P$ XG clauses translated from the grammar in figure 6. "The variables beginning with " $X$ " in the figure 8 are introduced to handle lefit extraposition. This variable is called $X$ list (extraposition list) which were intro. duced in XG [8]. Information pertaining to slash categories is pushed into the $X$ list and is then transferred from category to category during the analysis process. The predicate goal $x$ is an extended version of the predicate goal in the BUP system, which pops up the slash category from the $\dot{X}$ list when the trace is lourd. Note that variables for parse tree information, the names of which begin with "T", are antomatically inserted and that the representation of link information (in braces) is also modified.

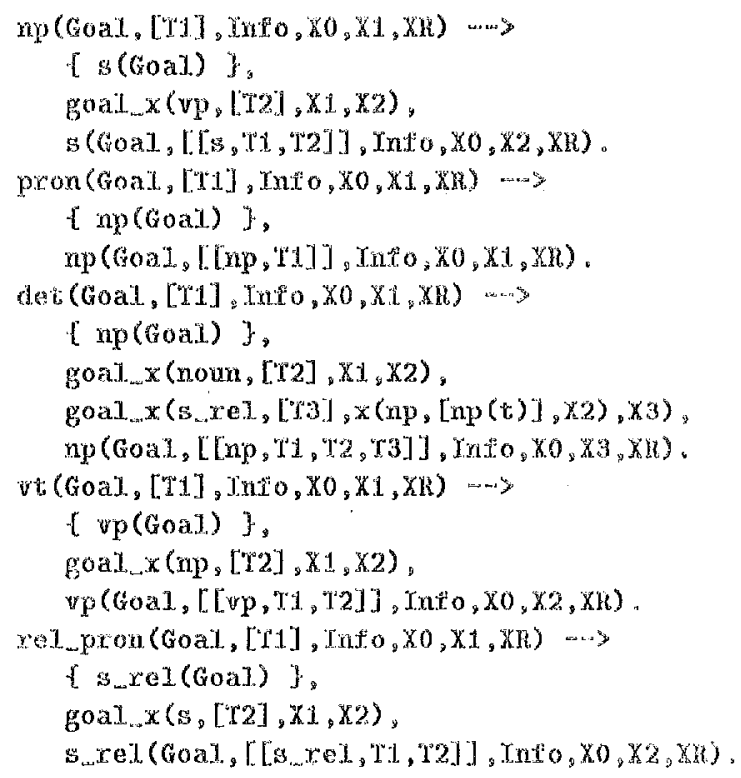

Figure 8: $130 \mathrm{P}-\mathrm{XC}$ clauses translated from tigure 6

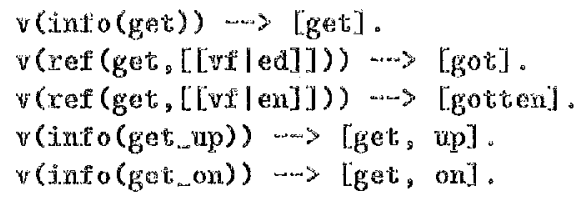

Figure 9: Saxnple dictionary including idioms

\section{\$ THET structwred dictiomary}

This chapter explains the TRre stuctured dictionary, another extension to the BUP. XG system and the BOP systern. The TRUE strmctnred dictionary requires less menory, provides laster dictionary reference and flexible idiom hay. dling.

\subsection{There structure}

The name "TRYI": is taken from "reTMIEval" [1] and it means a kind of tree structure. A dictionary written in DCG is translated into a TRIE strnctured dictionary by the TRIE dictionary translator. The TRIE structure is a tupple which has three elements, that is "word", "information for woid(s)" and "its child ThIS structure".

For example, the dictionary written in DCG shown in fig. ure 9 would be translated to the TRTF structured dictionary shown in ngure 10.

To look up a TRIS structured dictionary, the dichionary reference progran sewrches through the tree matching the input string with the first clement of the TKIE structure and, information for the string of inpu: is rexieved only after the last word of the input string is matched. Actually, the translator bundles up the dictionary extries which has the same first word into a clause (see how the entries "get", "get on". and "get up" are translated in figure 10). By wsire; this stmcture for the dictionary, the system can avoid the 


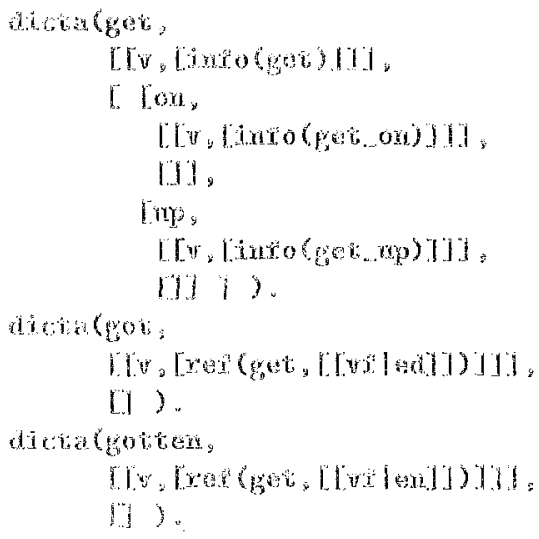

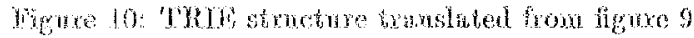

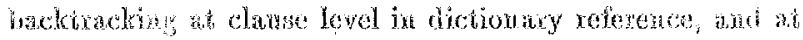

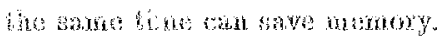

Ia tigure 9 , the argument of the hoad is the intomation of

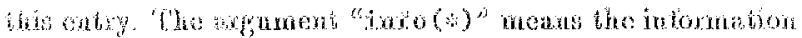

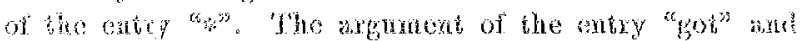

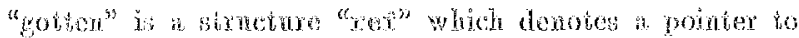

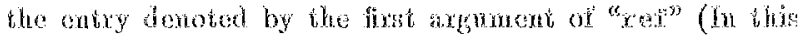

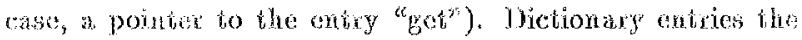
intomation of which anly aifow from each other partidly,

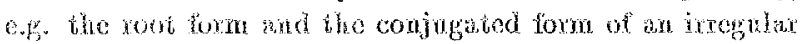

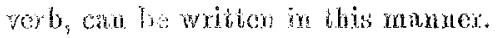

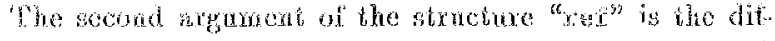

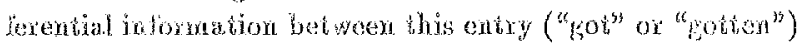

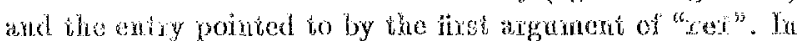

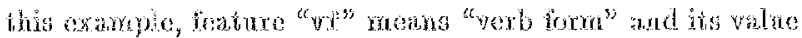
"od" and "cri" means "past" and "past jarticiplo" wospes. hivoly. With such a dempription, users do not have to wite

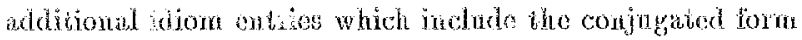
of ingyula verbs. In the canst of regular verbs, where cor-. jugated forms wo proessed by the nomhological analysis pograxh bult in the dichondey reference progrma, idiom enbies whin indude the conjugated fom are not noessary. For axame, wsers do not have to write the idiom entry "kicked the breket", if the entry "kicte the brokes" is wribing.

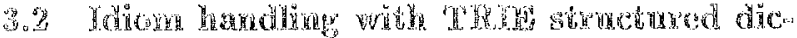 tidurary}

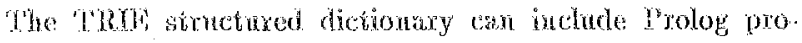

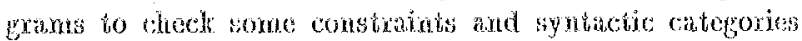
in its "wont" postion (firet alenext of inpplo). This teatre

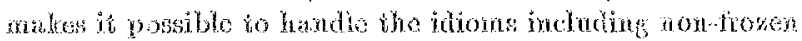

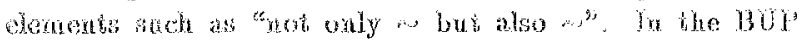
aystom and the BOP Yre system, illo system regads such

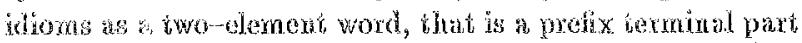

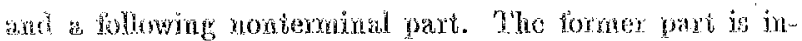

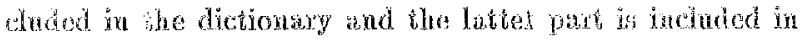

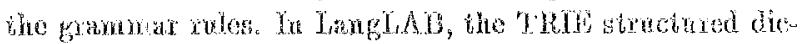

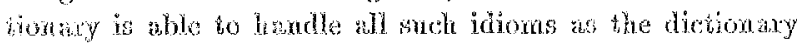

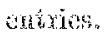

The idion ontes whoh inchde androzon elomention such

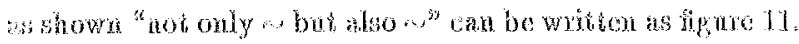

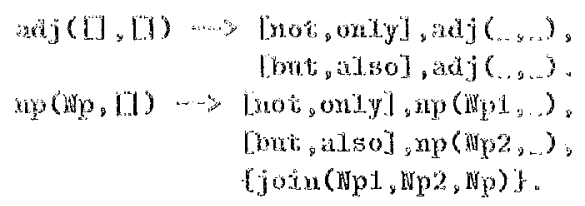

Thin at: Sample dictionary with nomterminal symbols and mograns in the rule body

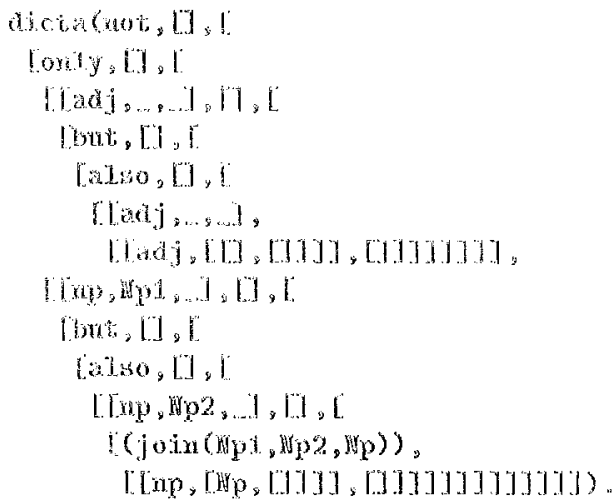

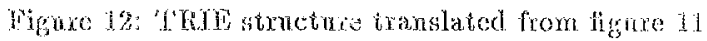

And figure $1 \%$ is the result of the transtation.

En the case of $B C G$, as the idion entry such as figme 11 is

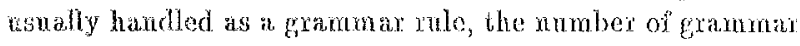
rules increases and ineficicncy of analysis process yesults, it is preterable to hande gramor tules and dictionary cotries aeparatcly.

A.s stown in figure 12, the translator converts the Prolog programs in the dictionary antry " $\{$ joins(Npt, ifp., inp) $\}$ " into the form "(join( Apl, inp?, thp))". The dictionary retarence program calls the program enclosed by parenthesis wher it cricowiens such a forn. In the same way, the synbitetic category in the dictionary entries such as "np $(10 \mathrm{p}, .$.$) "$ we converted into the list the first eloment of which is a category atome and the rest of which are arguments of the chefory (lny, Hon, 7). The dictionary reference program calls î̀ie predicate goul $($ goal $(n p, b y p i, \ldots, X, Y)$ ) for such. a. lorm.

The TRIS structured dictionary enables the langh $\Lambda \mathrm{B}$ syetem to handle idioms with versatility $\left[\begin{array}{l}d \\ ]\end{array}\right]$.

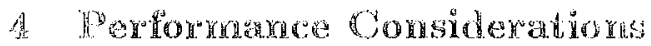

We conducted experiments to verify the effect of optimam.

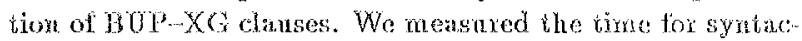
tic analysis of ten sample sentences. The experiment envj.roxment is as lollows:

6) Wachine : Sunas/260 Workstation

Prolog: Quintus Prolog Release 1.6

o Gramme : 168 rules in XOS

Ju the experionent, we measured the tirne required to ob iaim all perse tree before and after the optimization for each 
Table 1: Analysis time using interpretive code

\begin{tabular}{|r|r|r|r|r|r|} 
No. & $\begin{array}{r}\text { Number } \\
\text { of Words }\end{array}$ & $\begin{array}{r}\text { Number } \\
\text { of Trees }\end{array}$ & \multicolumn{2}{|c|}{ Analysis Time [msec] } & Ratio \\
\cline { 4 - 5 } & 1 & 9 & $80,4 \mathrm{~J} 5$ & 8,552 & 9.40 \\
2 & 14 & 9 & 12 & (2) LangLAB & $(12)$ \\
3 & 4 & 12 & 18,868 & 2,700 & 6.99 \\
4 & 3 & 7 & 46,700 & 4,983 & 9.37 \\
5 & 1 & 10 & 30,900 & 3,600 & 8.58 \\
6 & 3 & 11 & 39,634 & 4,050 & 9.79 \\
7 & 4 & 18 & 95,933 & 9,550 & 10.05 \\
8 & 9 & 21 & 323,167 & 26,183 & 12.34 \\
9 & 2 & 19 & 87,550 & 9,349 & 9.36 \\
10 & 4 & 17 & 180,300 & 15,816 & 11.40 \\
& 1 & 25 & 116,284 & 12,083 & 9.62 \\
\hline & & & & average & 9.69 \\
\hline
\end{tabular}

Table 2: Analysis time using compiled code

\begin{tabular}{|r|r|r|r|r|r|}
\hline No. & $\begin{array}{r}\text { Nurnber } \\
\text { of Words }\end{array}$ & $\begin{array}{r}\text { Number } \\
\text { of Trees }\end{array}$ & \multicolumn{2}{|c|}{ Analysis Time (1) BUP (1) XG } & Ratio \\
\hline 1 & 14 & 9 & 20,485 & 4,134 & 4.96 \\
2 & 4 & 12 & 2,467 & 1,299 & 1.90 \\
3 & 3 & 7 & 4,783 & 2,284 & 2.09 \\
4 & 1 & 10 & 2,884 & 1,566 & 1.84 \\
5 & 3 & 11 & 4,383 & 1,917 & 2.29 \\
6 & 4 & 18 & 18,768 & 4,500 & 4.17 \\
7 & 9 & 21 & 127,400 & 14,000 & 9.10 \\
8 & 2 & 19 & 13,450 & 4,450 & 3.02 \\
9 & 4 & 17 & 59,468 & 8,216 & 7.24 \\
10 & 1 & 25 & 23,650 & 5,801 & 4.08 \\
\hline & & & & average & 4.07 \\
\hline
\end{tabular}

sample sentence. 'This analysis does not include morphological analysis. Table 1 is the result of the experiment in the interpretive mode and table 2 is the one in the compiled mode. The fourth and the fifth column of the table is the time to an alyze the sentence in the original BUP-XG system and in the LangLAB system respectively. Time is shown in millisecond.

Results showed that in comparison to the original BUPXG system, the analysis sped up 10 times in the interpretive mode and 4 times in the compiled mode. The optimization is less effective in the compiled mode than in the interpretive mode. Ilowever, this optimization is practical because de bugging is usually done in the interpretive mode. We believe that LangLAB has the capacity for practical use.

There is a related work SAX [6] by Matsumoto. SAX is also a parsing system based on logic programming, but its parsing strategy is bottom-up and breadth-first. Okunishi of ICOT reports that Lang LAB is $6 \sim 10$ times faster than $\mathrm{SAX}$ in the interpretive mode. However, in the compiled mode, SAX is $6 \approx 16$ times faster than LangLAB [11]. SAX has still yet to be modified to handle idioms. If this modification is introduced, debugging can be done on langLAB in the interpretive mode and the debugged grammar can be executed on SAX in the compiled mode.

\section{Conclusion}

We have made the following modification to the original BOP $-X G$ :
- Optimized and enhanced translated code

- Adopted TRIE structured dictionary

With these modifications, the analysis sped up in comparison to the original BUP-XG system and flexible idiom handling became possible. We believe that LangLAB has become a more powerful and practical tool for natural language processing. We plan to develop a natural language processing system which includes semantic analysis, based on LangLAB.

\section{References}

[1] A. V. Aho, J. E. Hopcroft, and J. D. Ulluran. Dato Structures and Algorithms. Addison-Wesley, 1983.

[2] A. Colmerauer Metamorphosis grammar. In Natural Language Communication with Computers, pages 133190, Springer-Verlag, 1978.

[3] G. Gazdar and A. F. Pullum. Generalized Phrase Structure Grammar:A Theoretical Synopsis. Indiana University Linguistics Club, 1982.

[4] M. Gross. Lexicon-grammar: the representation of compound words. In COLING '86, pages 1-6, 1986.

[5] S. Konno and H. Tanaka. Processing left-extraposition in bottom up parsing system. Computer Software, $3(2): 115-125,1986$. (in Japanese).

[6] Y. Matsumoto and R. Sugimura. A parsing system based on logic programming. In $I J C A I$ ' 87 , pages $671-$ $674,1987$.

[7] M. McCord. Natural language processing in prolog. In Adrian Walker, editor, Knowledge Systems and Prolog, chapter 5, pages 291-402, Addison-Wesley, 1987.

[8] F. Pereira. Extraposition grammar. American Journal of Computational linguistics, 7(4):243-256, 1981.

[9] F. Pereira and D. Warren. Definite clause grammar for language analysis - a survey of the formalism and a comparison with augmented transition networks. Artificial Intelligence, 13(3):231-278, 1980.

[10] J.R. Ross. Constraints on variables in syntax. In $O_{n}$ Noam Chomsky: Critical Essays, Anchor Books, 1974.

[11] T. Okunishi, et.al. Comparison of logic programming based natural language parsing systems. In 2nd lnternational Workshop on Natural Language Understanding and Logic Programming, pages 90-102, 1987.

[12] T. Winograd. Language as a Cognitive Process. Volume 1:Syntax, Addison-Wesley, 1983.

[13] W.A. Woods. Experimental parsing system for transition network grammar. In Natural Language Process ing, Algorithmic Press, 1971.

[14] Y. Matsumoto, et.al. Bup:a bottom-up parser embedded in Prolog. New Generation Computing, I(2):145$158,1983$. 